Cite this: Phys. Chem. Chem. Phys., 2013, 15, 12451

Received 28th February 2013, Accepted 5th April 2013

DOI: $10.1039 / \mathrm{c} 3 \mathrm{cp} 50829 \mathrm{c}$

www.rsc.org/pccp

\title{
Polymorphism in porphyrin monolayers: the relation between adsorption configuration and molecular conformation $\dagger$
}

\begin{abstract}
Michiel J. J. Coenen, ${ }^{a}$ Duncan den Boer, ${ }^{a}$ Fieke J. van den Bruele, ${ }^{\text {a }}$ Thomas Habets, ${ }^{a}$ Koen A. A. M. Timmers, ${ }^{a}$ Minko van der Maas, ${ }^{a}$ Tony Khoury, ${ }^{b}$ Dwi Panduwinata, ${ }^{b}$ Maxwell J. Crossley, ${ }^{b}$ Jeffrey R. Reimers, ${ }^{b}$ Willem J. P. van Enckevort, ${ }^{a}$ Bas L. M. Hendriksen, ${ }^{a}$ Johannes A. A. W. Elemans ${ }^{\star a}$ and Sylvia Spellert ${ }^{a}$

Self-assembled monolayers of meso-5,10,15,20-tetrakis(undecyl)porphyrin copper(॥) on a graphite/ 1-octanoic acid interface have been studied by Scanning Tunnelling Microscopy. Four distinct polymorphs were observed, varying in their unit cell size. Arrays of unit cells of the various polymorphs seamlessly connect to each other via shared unit cell vectors. The monolayers are not commensurate, but coincident with the underlying graphite substrate. The seamless transition between the polymorphs is proposed to be the result of an adaptation of the molecular conformations in the polymorphs and at the boundaries, which is enabled by the conformational freedom of the alkyl tails of these molecules.
\end{abstract}

\section{Introduction}

The controlled bottom-up construction of self-assembled molecular monolayers is of paramount importance for the development of single molecule electronics, biosensors and catalysts. ${ }^{1}$ Despite the numerous studies on the self-assembly of molecules on surfaces, a complete understanding of the relationship between the structure of a molecule and the structure of the monolayers it forms on a given surface is still rather limited. One aspect of molecular selfassembly on surfaces that is receiving increasing attention is 2-dimensional (2D) polymorphism: the ability of one compound, or combination of compounds, to form several different 2D surface structures. $^{2,3}$ Scanning Tunnelling Microscopy (STM) ${ }^{4}$ provides an excellent tool to study the structure of self-assembled monolayers on solid surfaces, ${ }^{5}$ and STM studies of the existence of $2 \mathrm{D}$ polymorphs have been reported for small $^{6}$ and extended phthalocyanine derivatives, ${ }^{7}$ as well as for porphyrin-based macromolecules. ${ }^{8}$ For other types of macrocycles it was shown that molecules in different polymorphs can exhibit different properties.

\footnotetext{
${ }^{a}$ Radboud University Nijmegen, Institute for Molecules and Materials, Heyendaalseweg 135, 6525 AJ Nijmegen, The Netherlands. E-mail: J.Elemans@science.ru.nl

${ }^{b}$ The School of Chemistry, University of Sydney, NSW 2006, Australia

$\dagger$ Electronic supplementary information (ESI) available: Extra STM images showing accurate vector measurements, moiré patterns, graphite overlays, cross sections, and vector theory. See DOI: 10.1039/c3cp50829c

† Current address: University of Rostock, Germany.
}

De Feyter and co-workers revealed that, depending on the concentration, alkoxylated dehydrobenzo[12]annulene (DBA) derivatives can form either a close-packed linear polymorph or nanoporous networks at a solid/liquid interface. ${ }^{3}$ Such networks can be used to bind guest molecules, and the different polymorphs were found to induce different complexation behaviour of these guests. ${ }^{9}$ Burke et al. showed that the self-assembly into different polymorphs can affect the optoelectronic properties of 3,4,9,10perylenetetracarboxylic dianhydride (PTCDA) molecules on a $\mathrm{NaCl}(001)$ surface. $^{10}$

We here report STM studies of the polymorphism of porphyrin molecules at a solid/liquid interface. Porphyrins are interesting molecules for use in a wide range of applications, because of their rich photo-physical, electronic and chemical properties. They have been applied as catalysts, chemical sensors, photosensitisers, light emitting diodes, photovoltaic cells, molecular wires, transistors, and organic semiconductors. Organised arrays of porphyrins on a surface have been investigated with the help of STM, ${ }^{11}$ and studies range from those of simple porphyrins equipped with alkyl tails ${ }^{12}$ or carboxylic acids, ${ }^{13}$ to those of more complex covalently linked networks, ${ }^{14}$ rings ${ }^{15}$ and wheels ${ }^{16}$ consisting of tens of porphyrins.

We will discuss the self-assembly behaviour of the alkylfunctionalised meso-5,10,15,20-tetrakis(undecyl)porphyrin copper(II) ((TUP)Cu, Fig. 1) at a graphite/1-octanoic acid interface. The main observation that we will discuss is the sharing of unit cell vectors of the 2D polymorph structures this compound forms, and the implications of this sharing on the adsorption configurations of 


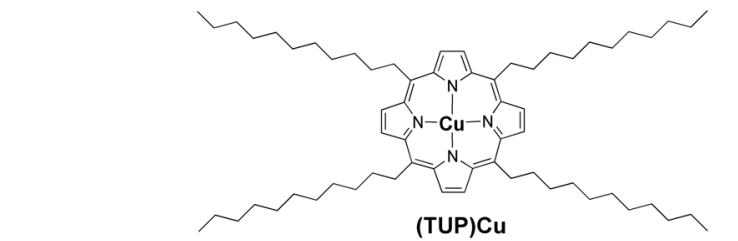

Fig. 1 Molecular structure of meso-5,10,15,20-tetrakis(undecyl)-porphyrin copper(II) ((TUP)Cu).

the porphyrin molecules in the different polymorphs. We will provide a rational explanation for this behaviour in terms of the molecular conformations of the adsorbates.

\section{Results and discussion}

\section{$2.12 \mathrm{D}$ porphyrin polymorphs}

At the graphite/1-octanoic acid interface, (TUP)Cu self-assembles into monolayers in which four distinct unit cells can be distinguished (Fig. 2a-d). ${ }^{17}$ The porphyrin cores in the STM images appear as either disk-like (Fig. 2a), square-like (Fig. 2b) or as four-lobed structures (Fig. 2c and d). These differences can be caused by variations in planarity of the porphyrin aromatic planes and/or in the sharpness of the STM tip. The alkyl tails appear darker and are generally less clearly visualised. Because of the differences in unit cell areas of the polymorphs, we refer to them as "large" (L), "border" (B), "medium" (M), and "small" (S). The unit cell parameters are summarised in Table 1. All four unit cells can coexist at the interface of graphite and 1-octanoic acid. In Fig. 2e, the lower lying terrace is covered with the L polymorph, while the upper terrace is mainly covered with patches of the $\mathrm{M}$ polymorph which are occasionally separated by straight rows of B unit cells. We reported previously that the $\mathrm{M}$ and $\mathrm{B}$ unit cells can coexist in virtually any ratio on a given terrace at the graphite/1-octanoic acid, and we also discussed the

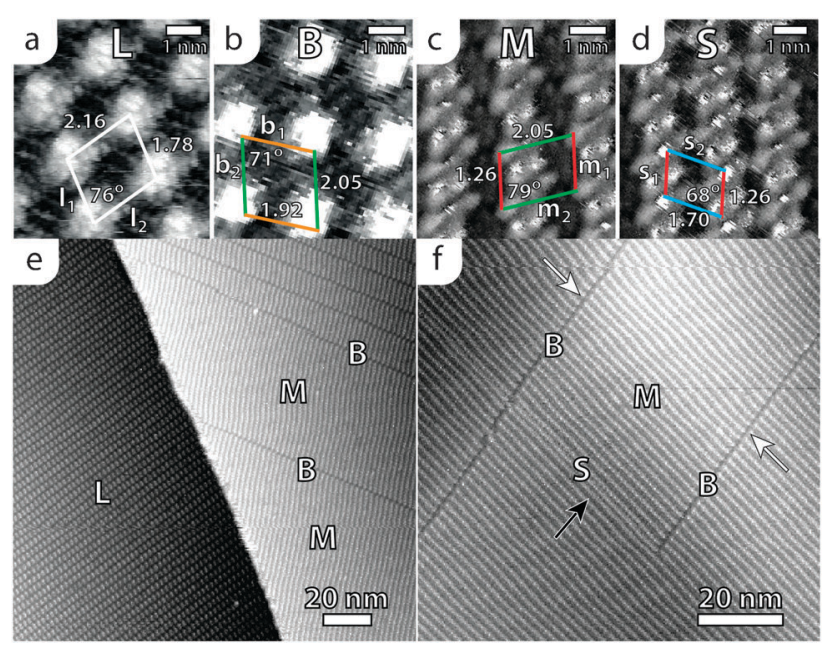

Fig. 2 (a-d) STM topography images of the four main polymorph structures of (TUP)Cu (L, B, M and S) found at the graphite/1-octanoic acid interface; unit cell vectors are drawn in; image parameters: (a) $V_{\text {bias }}=470 \mathrm{mV}, I_{\text {set }}=13 \mathrm{pA}$; (b) $V_{\text {bias }}=$ $-710 \mathrm{mV}$, $I_{\text {set }}=15 \mathrm{pA}$; (c) $V_{\text {bias }}=-710 \mathrm{mV}$, $I_{\text {set }}=15 \mathrm{pA}$; (d) $V_{\text {bias }}=-710 \mathrm{mV}$, $I_{\text {set }}=$ $15 \mathrm{pA}$; (e and f) overview images. Image parameters: (e) $V_{\text {bias }}=-750 \mathrm{mV}, I_{\text {set }}=7 \mathrm{pA}$; (f) $V_{\text {bias }}=-710 \mathrm{mV}, I_{\text {set }}=14 \mathrm{pA}$. See text for explanation of the arrows.
Table 1 Unit cell vectors and angles of the various polymorphs ( $L, B, M$, and $S$ ) found for (TUP)Cu at the graphite/1-octanoic acid interface

\begin{tabular}{lllll}
\hline Unit cell & Unit cell area $\left(\mathrm{nm}^{2}\right)$ & Unit cell vector & Size $(\mathrm{nm})$ & Angle $\left(^{\circ}\right)$ \\
\hline $\mathrm{L}$ & $3.65 \pm 0.30$ & $\boldsymbol{l}_{1}$ & $1.74 \pm 0.08$ & $76 \pm 5$ \\
& & $\boldsymbol{l}_{2}$ & $2.16 \pm 0.14$ & \\
$\mathrm{~B}$ & $3.72 \pm 0.26$ & $\boldsymbol{b}_{1}$ & $1.92 \pm 0.09$ & $71 \pm 4$ \\
& & $\boldsymbol{b}_{2}=\boldsymbol{m}_{2}$ & $2.05 \pm 0.05$ & \\
$\mathrm{M}$ & $2.54 \pm 0.12$ & $\boldsymbol{m}_{1}=\boldsymbol{s}_{1}$ & $1.26 \pm 0.05$ & $79 \pm 4$ \\
$\mathrm{~S}$ & $2.07 \pm 0.16$ & $\boldsymbol{s}_{2}$ & $1.70 \pm 0.15$ & $68 \pm 4$ \\
\hline
\end{tabular}

dynamics of these mixed domains of $\mathrm{M}$ and $\mathrm{B}$ unit cells. ${ }^{17}$ At first sight, the B surface structure looks very similar to the $\mathrm{L}$ polymorph, but it is different both in terms of unit cell parameters and occurrence. It occurs in most occasions as a straight border between different patches of the M polymorph (Fig. 2e and $\mathrm{f}$ ), and it can be considered as a (anti-phase) boundary structure. However, because we occasionally observed it as a 2D structure we treat B as an individual polymorph. The STM image depicted in Fig. 2f shows that besides the $B$ and $\mathbf{M}$ polymorphs, the $\mathrm{S}$ surface structure can also coexist on the same terrace at the graphite/1-octanoic acid interface.

Like the B unit cells, the unit cells of the $S$ polymorph can also be mixed with those of the $\mathbf{M}$ polymorph in a highly ordered fashion. The area in Fig. 2f labelled "M" ends with sharp borders of B unit cells in the upper left and lower right (white arrows), while in the centre of the image it is terminated by an equally straight patch of the $S$ polymorph (black arrow).

Higher concentrations of (TUP)Cu in the supernatant solution generally give rise to denser packed polymorphs at the surface: when [(TUP)Cu] $=10^{-7} \mathrm{M}$, mainly the L polymorph is formed, while at a concentration of $10^{-4} \mathrm{M}$ mostly $\mathrm{M}$ is formed, and at a concentration of $>10^{-3} \mathrm{M}$ mostly $\mathrm{S}$. This concentration dependency is in line with previous studies of De Feyter on the behaviour of DBA derivatives. ${ }^{3}$ However, we recently reported that in the case of the (TUP)Cu polymorphs the monolayers are not always in thermodynamic equilibrium with the supernatant solution. ${ }^{17}$ This means that the distribution of the polymorphs at the surface can vary significantly, depending on local kinetic trapping. We will discuss this complex interplay between thermodynamics and kinetics more extensively in a forthcoming paper.

\subsection{Shared unit cell vectors and adsorption configurations}

The STM images in Fig. 2e and f show an interesting property of the polymorphs formed by (TUP)Cu: they connect seamlessly. This is caused by the fact that the M, B and $S$ unit cells share unit cell vectors among each other. The B and $\mathrm{M}$ unit cells, for instance, share their longest unit cell vectors, $\boldsymbol{b}_{2}=\boldsymbol{m}_{2}$, while the second unit cell vector of the $\mathbf{M}$ polymorph is shared with a vector of the $\mathrm{S}$ polymorph, $\boldsymbol{m}_{1}=\boldsymbol{s}_{1}$.

The equality of the $\boldsymbol{m}_{2}$ and $\boldsymbol{b}_{2}$ vectors was determined with high accuracy by a direct, relative comparison (Fig. 3) and the numerical values of the vector length and angle were determined by comparison to the underlying graphite lattice (Fig. S5, ESI $\dagger$ ). The red dashed line on the left side of the STM image in Fig. 3 connects the centres of a row of (TUP)Cu adsorbates. 


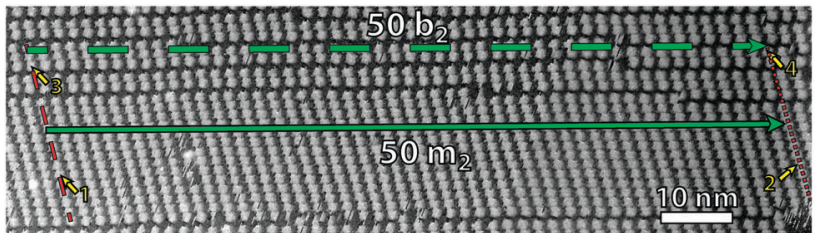

Fig. 3 STM topography image of a self-assembled monolayer of (TUP)Cu at the graphite/1-octanoic acid interface, showing the connection between $\mathrm{M}$ and $\mathrm{B}$ polymorphs. $V_{\text {bias }}=-780 \mathrm{mV}, I_{\text {set }}=8 \mathrm{pA}$. See the text for further explanation.

It passes through the centre of the adsorbate marked " 1 " in the middle of the patch of the $\mathrm{M}$ polymorph, and also through the centre of the porphyrin marked " 3 " at the border formed by a row of B unit cells. The red dotted line on the right of the image passes through a similar row of (TUP)Cu adsorbates, in M, "2", and in B, "4". The solid green arrow extends from the red dashed line on the left to the red dotted line on the right, and has a length of $50 m_{2}$ unit cell vectors, which we determined simply by counting. The green dashed line through $\mathrm{B}$ has the same length and corresponds to $50 \boldsymbol{b}_{2}$ unit cell vectors. Over a translation of 50 unit cell vectors no apparent phase shift is present between the molecules within the $\mathbf{M}$ polymorph ("1","2") and those along the B border ("3","4"). The same type of measurement was used to assess the equality of the $\boldsymbol{m}_{1}$ and $\boldsymbol{s}_{1}$ unit cell vectors (Fig. S2a, ESI + ). The observation that the vectors of the $\mathrm{M}, \mathrm{B}$ and $\mathrm{S}$ unit cells do not show a measurable loss of registry over 50 unit cells puts the maximum difference between the $\boldsymbol{b}_{2}$ and $\boldsymbol{m}_{2}$ vectors, as well as between the $\boldsymbol{m}_{1}$ and $\boldsymbol{s}_{2}$ vectors, on the order of a few picometer.

Because of the unit cell vector sharing, a (TUP)Cu molecule at the boundary of two polymorphs can simultaneously be part of the unit cells of two different polymorphs. This is illustrated in Fig. 4. In the STM image (Fig. 4a), different patches of the B, $\mathrm{M}$ and $\mathrm{S}$ polymorphs meet. The different unit cell vectors are drawn in different colours $\left(\boldsymbol{s}_{2}\right.$ in blue, $\boldsymbol{s}_{1}=\boldsymbol{m}_{1}$ in red, $\boldsymbol{m}_{2}=\boldsymbol{b}_{2}$ in green, and $\boldsymbol{b}_{1}$ in orange). Some of the shared unit cell vectors are part of two different unit cells at the same time. The $\boldsymbol{m}_{2}=\boldsymbol{b}_{2}$ vector, marked by the yellow arrow, is for instance part of an $\mathbf{M}$ unit cell on one side, while it is part of a B unit cell on the other side. Similarly, the $\boldsymbol{m}_{1}=\boldsymbol{s}_{1}$ unit cell vector, marked by the purple arrow, is simultaneously part of an $\mathrm{M}$ and an $\mathrm{S}$ unit cell. To emphasise the fact that this vector is not always necessarily part of both these different unit cells, the white arrow in Fig. 4a marks an $\boldsymbol{m}_{\mathbf{1}}=\boldsymbol{s}_{1}$ unit cell vector that is fully surrounded by unit cells of the $\mathrm{S}$ polymorph, and the black arrow marks one that is enclosed by only $\mathrm{M}$ unit cells. To make a connection between the unit cell vectors and the conformational symmetry of the (TUP)Cu adsorbates, we will use the term adsorption configuration to refer to the collection of relative locations where the direct neighbours of a given (TUP)Cu adsorbate are found. In Fig. $4 \mathrm{~b}$ the part of the lattice in Fig. 4a surrounding the molecule marked $\mathcal{M S}$ is depicted. A molecule in this configuration has eight direct neighbours (1-8). The thick, coloured, lines connect the central $\mathcal{M S}$ adsorbate to four of its neighbours $(2,4,6,8)$. We represent the molecules in the various adsorption configurations by a square in which each of the four

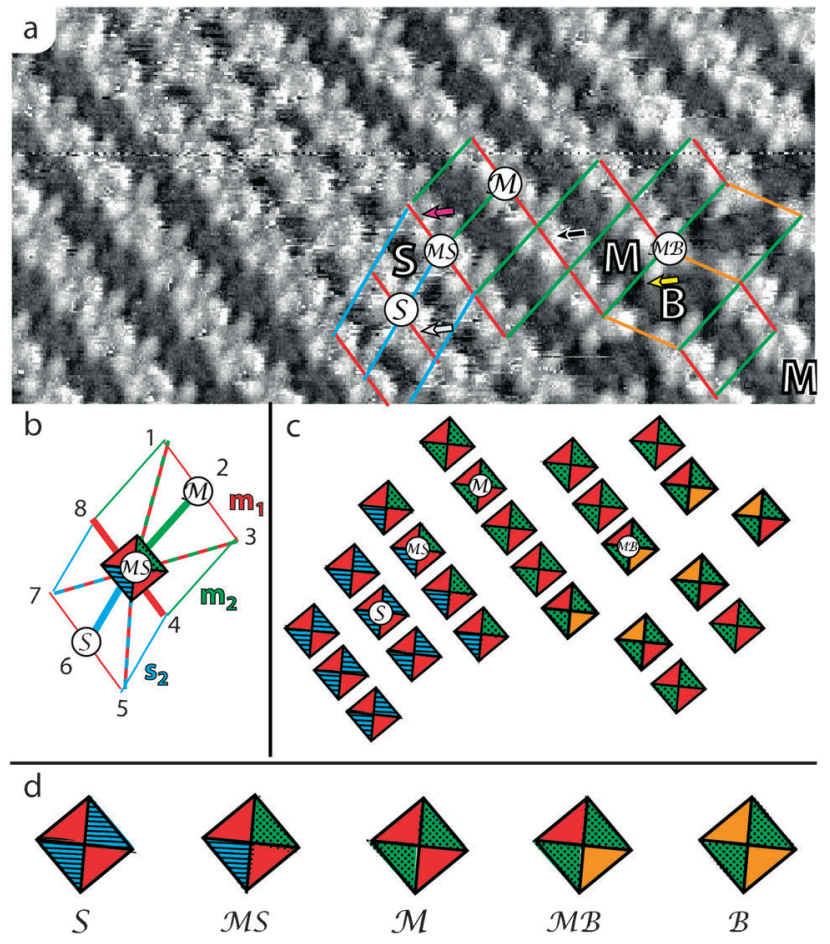

Fig. 4 (a) STM topography image of a monolayer of (TUP)Cu at the graphite/ 1-octanoic acid interface, illustrating that the $\mathrm{M}$ and $\mathrm{S}$ polymorphs share one of their unit cell vectors $\left(\boldsymbol{m}_{1}=\boldsymbol{s}_{1}\right.$, drawn in red) as well as the $\mathrm{M}$ and $\mathrm{B}$ polymorphs $\left(\boldsymbol{m}_{2}=\boldsymbol{b}_{2}\right.$, drawn in green); see text for explanation of the arrows. (b) Schematic overview of the adsorption configuration of the adsorbate marked $\mathcal{M S}$ in (a). (c) Schematic representation of part of the adsorbates in (a) using the squares representing the adsorption configurations of (TUP)Cu, an overview of which is given in (d). See the text for further explanation.

quadrants is coloured according to these unit cell vectors. For the $\mathcal{M S}$ adsorption configuration the square therefore has one blue, one green and two red quadrants. The colours of these four quadrants also define the locations of the other four neighbours $(1,3,5,7)$ since these unit cell vectors obey the same algebraic rules as normal overlayer lattices. This is indicated by the dashed bi-coloured lines for $\mathcal{M S}$ in Fig. $4 \mathrm{~b}$, and is also true for the other adsorption configurations. A more detailed description is given in Fig. S6 (ESI + ). In Fig. 4d the representing squares for (TUP)Cu adsorbates in the $\mathcal{S}$, $\mathcal{M S}, \mathcal{M}, \mathcal{M B}$ and $\mathcal{B}$ configurations are drawn, and from these squares we can easily see that shared unit cell vectors lead to partially shared configurations. The representing squares of the porphyrins adsorbed at the vertices of the grid sketched in Fig. 4a are depicted in Fig. 4c. The same orientation of the porphyrin cores in all polymorphs suggests that the conformations of and the interactions between the alkyl tails of the adsorbates determine the unit cell parameters. To further test this hypothesis we first investigated the interaction between the porphyrin monolayer and the underlying graphite surface in more detail.

\subsection{Interactions between the porphyrins and the graphite substrate}

To address whether the shared unit cell vectors and resulting shared adsorption configurations of the polymorphs are indeed 
dictated by intermolecular interactions between the molecules of (TUP)Cu, or that they are a direct result of commensurability of the overlayer with the underlying graphite substrate, we investigated the lattice relation between the graphite and the polymorphs. The precision of the unit cell coefficients, which we determined from STM images (Table 1), is not sufficient to unambiguously determine if they are integer multiples of the graphite lattice vectors. However, the frequent observation of moiré patterns in the STM images of all polymorphs (e.g. Fig. 5 and Fig. S3, ESI $\dagger$ ) indicates that they are not commensurate with the underlying graphite surface. ${ }^{18}$ As an example, in the moiré pattern of the $\mathrm{M}$ polymorph (Fig. 5b), molecules of equal apparent height are found after translating over $7 \boldsymbol{m}_{1}$ or $8 \boldsymbol{m}_{2}$. The moire patterns are caused by the fact that the molecules are adsorbed at slightly different locations of the unit cell of the underlying graphite substrate, thereby causing a slight periodic variation in the electronic coupling between the molecules and the graphite. This results in a periodic variation in the apparent height in STM images of such an overlayer. We should note that the moiré patterns were not always observed in the STM images, as their visibility seemed to depend on the tunnelling parameters and the atomic structure of the apex of the STM tip.

\subsection{Shared molecular conformations}

We will now relate the adsorption configurations of the (TUP)Cu molecules in the various polymorphs to the conformational properties of the alkyl chains of the molecules. A high resolution STM image of (TUP)Cu in the L polymorph, together with the proposed monolayer structure at the surface, is shown in Fig. 6. The molecular conformation, with interdigitated alkyl chains, is highly similar to the one found by Chin et al. for the related free base meso-tetrakis(nonadecyl)porphyrin ((TNP)2H). ${ }^{19}$ Based on their STM measurements and supported by Density Functional Theory (DFT) calculations, they proposed that the alkyl tails were slightly kinked upwards from the porphyrin ring, and that the bright lobes in the STM images not only include the porphyrin macrocycle but also the kinked portion of the tails. This interpretation is in line with the STM data we found for the L polymorph of (TUP)Cu. All four alkyl chains are aligned to the graphite $<11-20>$ directions, as do simple linear alkanes. ${ }^{20}$

The $\mathrm{B}, \mathrm{S}$ and $\mathrm{M}$ polymorphs share unit cell vectors and therefore we propose that also the molecular conformations

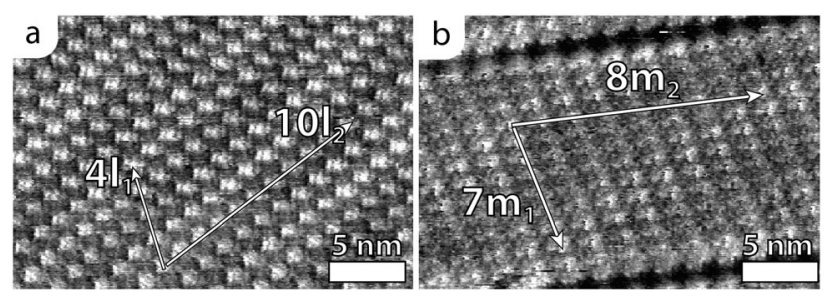

Fig. 5 STM topography images of monolayers of (TUP)Cu at the graphite/1 octanoic interface, which reveal the occurrence of moiré patterns in (a) the L polymorph and (b) the $\mathrm{M}$ polymorph. The periodicity of the superstructure is depicted along the unit cell vectors of the three polymorphs. (a) $V_{\text {bias }}=740 \mathrm{mV}$, $I_{\text {set }}=7 \mathrm{pA}$; (b) $V_{\text {bias }}=210 \mathrm{mV}$; $I_{\text {set }}=5 \mathrm{pA}$.

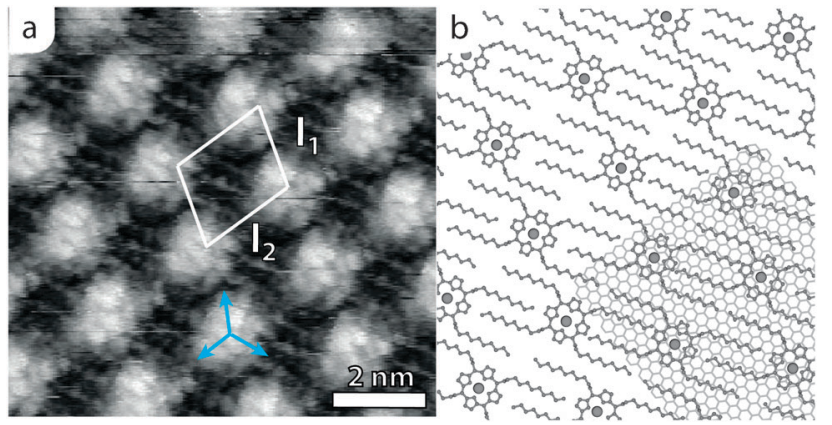

Fig. 6 (a) High resolution STM topography image of the L polymorph formed by (TUP)Cu at the graphite/1-octanoic acid interface; the unit cell vectors of the monolayer are drawn in white; $V_{\text {bias }}=450 \mathrm{mV}$, $I_{\text {set }}=12 \mathrm{pA}$; Blue arrows indicate graphite $<1 \quad 1-20>$ directions. (b) Proposed molecular conformations in this polymorph drawn as molecular models on top of part of a graphite lattice.

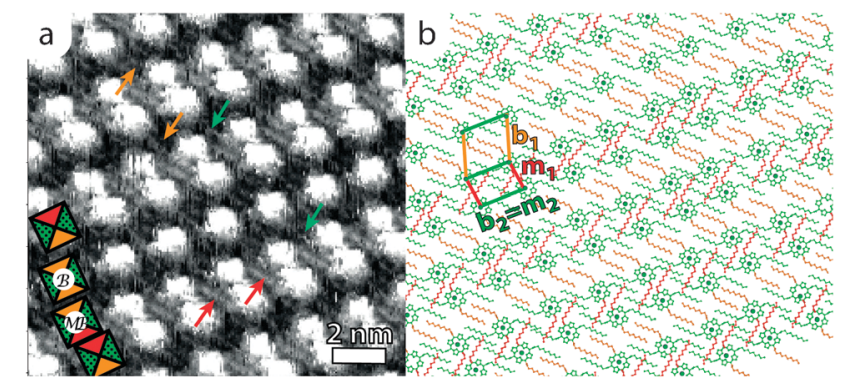

Fig. 7 (a) STM topography image of a monolayer of (TUP)Cu at the graphite/ 1 -octanoic acid interface, in which the molecules are adsorbed in $\mathcal{B}$ and $\mathcal{M B}$ adsorption configurations. Some schematic squares coding for the adsorption configurations are drawn in. Orange and green arrows point at alkyl chains adsorbed at the graphite between molecules in the $\mathcal{B}$ adsorption configuration. Red arrows point at higher appearing alkyl tails. $V_{\text {bias }}=-760 \mathrm{mV}, I_{\text {set }}=25 \mathrm{pA}$. (b) Proposed molecular conformations based on the STM image and the B and M unit cells with their unit cell vectors. See text for explanation.

within these polymorphs are related. Fig. 7 shows a high resolution STM image and the proposed molecular conformations for (TUP)Cu molecules in a domain in which the B and $\mathbf{M}$ polymorphs coexist.

We now assume that the molecular conformation of a (TUP)Cu molecule in a $\mathcal{B}$ adsorption configuration is similar to that of a molecule in the $\mathrm{L}$ polymorph. This assumption is supported by the fact that the $\mathrm{L}$ and $\mathrm{B}$ unit cells have comparable unit cell vectors and similar surface areas of $3.65 \pm$ $0.30 \mathrm{~nm}^{2}$ and $3.72 \pm 0.26 \mathrm{~nm}^{2}$, respectively. This suggest that also in the B polymorph there is enough space for all alkyl tails to be largely extended and adsorbed directly on the graphite surface. In the STM image in Fig. 7a, all four alkyl tails of (TUP)Cu molecules in the $\mathcal{B}$ adsorption configuration (indicated by green and orange arrows) appear to be extended along the same $<1 \quad 1-20>$ graphite direction.

In going from the $\mathrm{B}$ to the $\mathrm{M}$ and $\mathrm{S}$ polymorph the surface coverage of (TUP)Cu molecules increases and the layers compress, which leaves less and less space for the alkyl tails. Consider two (TUP)Cu molecules in the $\mathcal{B}$ configuration (Fig. 7). Moving these molecules closer together in the $\boldsymbol{b}_{1}$ direction results in the shorter 
$\boldsymbol{m}_{1}$ vector, and each molecule is now partly in B and partly in $\mathbf{M}$ (denoted $\mathcal{M B}$ in Fig. 7a). The alkyl tails that were originally adsorbed on the graphite in this gap (one of each molecule) now have to adjust to the reduced space and will have to adopt a new conformation (indicated by the red quadrant of the square). The three other alkyl tails can keep their original conformation (two green, one orange). Because a molecule of (TUP)Cu in an $\mathcal{M B}$ configuration has the locations of three of its four closest neighbours in common with a (TUP)Cu molecule in the B polymorph, we suggest that it will also have three of its four alkyl tails adsorbed in the same conformation as in $\mathcal{B}$ (Fig. 7b): the green-coloured and one of the orange-coloured tails. The fourth tail, which is coloured red in Fig. 7b, has only options to desorb and point into the liquid or to fold over on top of the green-coloured tails. The regions in the STM image where the tails should overlap (red arrows) indeed appear somewhat higher than the regions where only tails are found that are directly adsorbed to the graphite, which suggests the overlay of green and red tails (see also the cross section in Fig. S4, ESI $\dagger$ ). The formation of alkane bilayers has already been observed for long $n$-alkane molecules, ${ }^{21}$ and it was found that the apparent height of such a bilayer in STM measurements can be indistinguishable from that of an alkane monolayer. Also in templating studies, superimposed alkane chains have been proposed. ${ }^{22,23}$

The proposed relationship between the unit cells and the molecular conformations extends beyond the $\mathcal{M B}$ and $\mathcal{B}$ adsorption configurations: when (TUP)Cu molecules in an $\mathcal{M B}$ configuration are compared to molecules in an $\mathcal{M}$ configuration, again the molecular conformations of three of the four tails can be conserved, while the conformation of the fourth tail has to adapt to the compressed unit cell. A high resolution STM image of an $\mathbf{M}$ domain and the proposed molecular models of (TUP)Cu in the $\mathcal{M}$ adsorption configuration is shown in Fig. 8. In the model in Fig. $8 \mathrm{~b}$ the two green-coloured tails of each molecule are adsorbed in a similar fashion to the greencoloured tails of (TUP)Cu in the $\mathcal{B}$ and $\mathcal{M B}$ configurations in Fig. $7 b$, which explains the observed shared $\boldsymbol{m}_{2}=\boldsymbol{b}_{2}$ unit cell vector. The red-coloured tails are proposed to be adsorbed

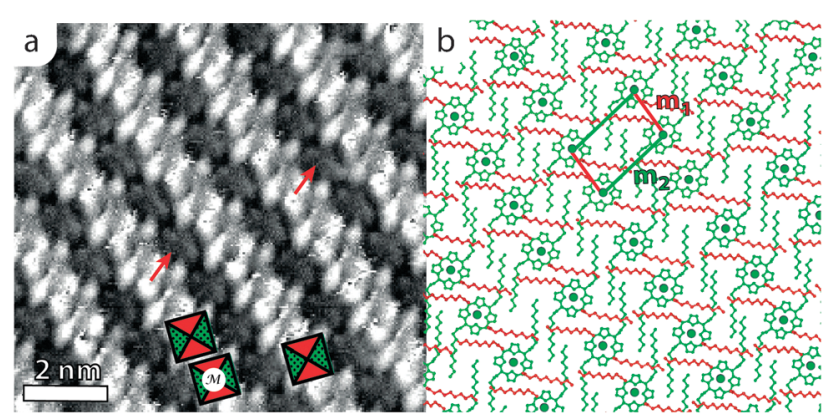

Fig. 8 (a) High resolution STM topography image of a domain of the $M$ polymorph formed by (TUP)Cu at the graphite/1-octanoic acid interface with some schematic adsorption configurations drawn in. Red arrows point at apparently overlapping alkyl tails. $V_{\text {bias }}=-710 \mathrm{mV}$, $I_{\text {set }}=13$ pA. (b) Proposed molecular conformations based on the STM image. A unit cell is drawn in. See text for explanation. similar to those of molecules in the $\mathcal{M B}$ configuration, i.e. overlapping with other alkyl tails, as is also suggested from the STM image (red arrows in Fig. 8a). The folding and adsorption on top of each other is not unlikely. We must note that the exact direction in which the second layer of alkyl tails is adsorbed is almost impossible to resolve from the STM images in Fig. 8a. We have therefore tentatively drawn the red-coloured tails in Fig. $8 \mathrm{~b}$ such that their connection to the porphyrin ring is rotationally equivalent by a four-fold rotation to the conformation of the green-coloured tails.

We now turn back to the STM image first presented in Fig. 4a, because it depicts a region where all three polymorphs that share unit cell vectors are observed with submolecular resolution. This STM image is shown again in Fig. 9a, now including the squares with the coloured quadrants to indicate the different adsorption configurations. In this image, porphyrin adsorbates in the $\mathcal{M B}, \mathcal{M}, \mathcal{M S}$ and $\mathcal{S}$ configurations can be observed in the same monolayer region. The molecular conformations of our model of part of the (TUP)Cu molecules in the STM image are sketched in Fig. 9b. Some of the alkyl tails of adsorbates in the $\mathcal{M B}$ and $\mathcal{M}$ adsorption configurations can be

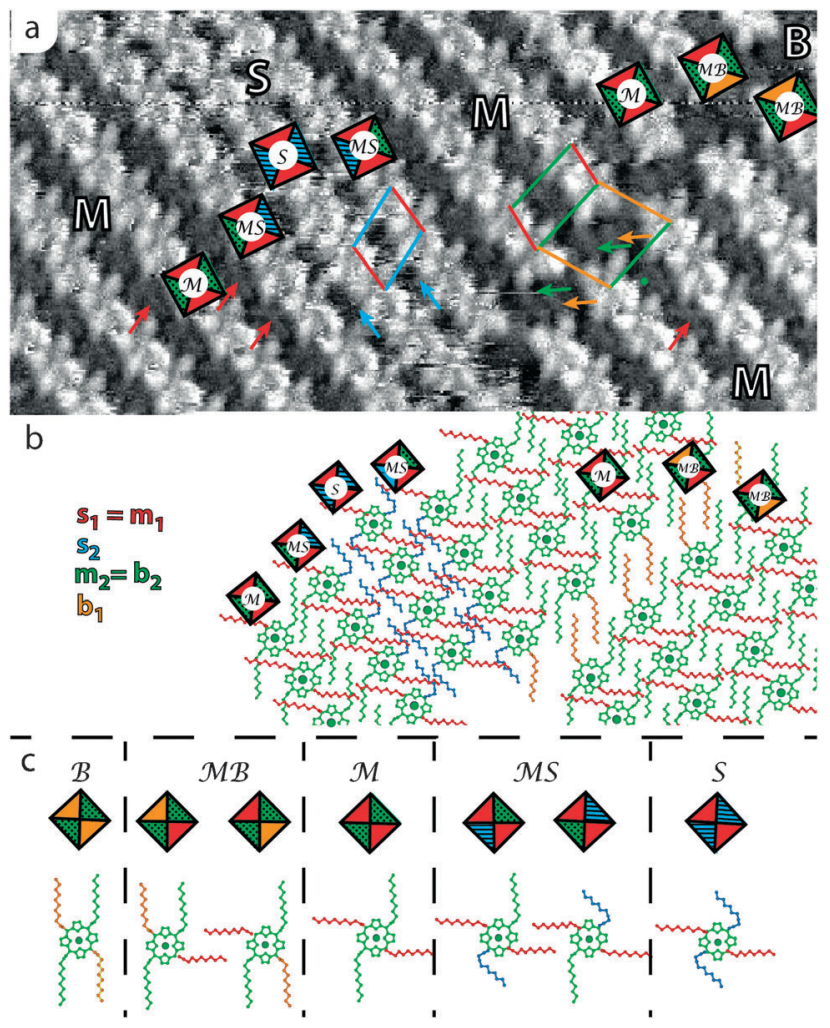

Fig. 9 (a) STM topography image in which molecules in the $M, B$ and $S$ polymorphs can be observed. $V_{\text {bias }}=-710 \mathrm{mV}, I_{\text {set }}=14 \mathrm{pA}, 12 \times 23 \mathrm{~nm}^{2}$. (b) Corresponding drawing of the proposed molecular structure of part of this monolayer. Some adsorption configurations of representative molecules are indicated at the same locations on both the STM image and the drawing. Unit cell vectors are drawn in, and regions in which the alkyl tails can be most easily seen in the STM image are indicated by small arrows with colours corresponding to the colours used for different tails in the drawing. (c) Overview of all 5 different adsorption configurations encountered for (TUP)Cu and their corresponding proposed molecular conformations. 
distinguished in the STM image. In the B unit cells, some all-trans oriented alkyl chains are visible (indicated by orange and green arrows). Exemplary alkyl chains that are proposed to be adsorbed on top of neighbouring tails are marked by red arrows.

The resolution of our STM images was not sufficient to suggest a conformation for the alkyl tails in the $\mathcal{M S}$ and $\mathcal{M}$ configuration. Since the $\mathcal{M S}$ configuration is for three quarters the same as the $\mathcal{M}$ configuration, we again propose that three of the four alkyl tails of a (TUP)Cu adsorbate in the $\mathcal{M S}$ configuration have the same conformations as those of an adsorbate in the $\mathcal{M}$ configuration. In this model, the fourth tail, indicated in blue, remains adsorbed to the graphite substrate in a bent conformation. In the $\mathcal{M S}$ adsorption configuration, only one tail assumes this conformation, while in the $\mathcal{S}$ configuration, the second tail also changes to this bent conformation. We propose that all four aliphatic tails remain adsorbed, instead of pointing in the supernatant solution, with two of them adsorbed on top of the other molecules rather than directly on the graphite surface (red-coloured in Fig. 9b and c).

The necessity of the blue-coloured tails to bend in the plane of the surface is caused by the lack of surface area available for them to adopt a fully extended all-trans conformation, as in the case of the $\mathbf{M}$ and $\mathrm{B}$ polymorphs. Such strongly bent aliphatic chain conformations are expected to be unfavourable in terms of molecular adsorption energy, but it is the adsorption energy per unit of surface area that is expected to be decisive for the formation of different surface structures. ${ }^{3}$ Because the surface density of the S polymorph is $23 \pm 10 \%$ higher than that of the $M$ polymorph, the difference in surface free energy per unit area between these two polymorphs will be less than the difference in molecular adsorption energy caused by a less favourable molecular conformation of the tails in the $\mathrm{S}$ polymorph. The occurrence of bent alkyl tails has already been proposed for other molecules self-assembled on graphite. ${ }^{23,24}$ The relationship between the five stable adsorption configurations and the corresponding molecular conformations of molecules of (TUP) Cu in domains in which $\mathrm{B}, \mathrm{M}$ and $\mathrm{S}$ coexist $(\mathcal{B}, \mathcal{M B}, \mathcal{M}$, $\mathcal{M S}, \mathcal{S}$ ), and their relationship to the other adsorption configurations are summarised in Fig. 9c. In every step on going from one configuration to the next, three tails are thus proposed to retain their conformation, while the conformation of the fourth tail changes in correspondence with the change in adsorption configuration. The structural model and the molecular conformation are mostly based on space and symmetry arguments. DFT modelling will be required to provide a more refined structural model.

\subsection{Discussion}

We argued that the partial similarity of molecular conformations of molecules of (TUP)Cu in adjacent polymorphs underlies the observed shared unit cell vectors. Even if details of the precise molecular conformations are slightly different than proposed, the underlying rationale remains valid: (TUP)Cu can self-assemble into different polymorphs because of the conformational freedom of the alkyl tails in the adsorption process, and the sharing of the unit cell vectors between the different polymorphs is a result of partially shared molecular conformations. This does not imply that the conformation of a given alkyl tail is solely responsible for the length of a unit cell vector and the angle with respect to the other unit cell vectors; the unit cell vectors arise from a complex interplay of forces between an adsorbed molecule and all its direct neighbours, and between the molecule and the underlying graphite. This could be addressed by extensive molecular modelling and expansion of the STM experiments to include meso-tetra-alkyl porphyrin derivatives in which one or more of the alkyl tails are replaced with alkyl tails with a shorter length. The formation of different polymorphs among which the conformation of the aliphatic tails of alkyl-functionalised molecules differs, has also been reported for hexakis(n-dodecyl)-peri-hexabenzocoronene $\left(\right.$ HBC-C $\left.{ }_{12}\right)$ on a templating monolayer of $n-\mathrm{C}_{50} \mathrm{H}_{102}$ at the graphite $/ n$-tetradecane interface. ${ }^{23}$ In that case, three different templated adlayer structures were formed on the same templating layer. Tahara et $a l^{24}$ reported the formation of five different polymorphs by alkyl and alkoxy-functionalised bis(dehydrobenzo[12]annulene) (bis-DBA) derivatives at the graphite/1,2,4-trichlorobenzene interface, also resulting from conformational freedom of the alkyl chains. Although both these studies do not explicitly report the presence of shared unit cell vectors, or the underlying shared molecular conformations, the STM images of that work contain these two elements. Hill et $a l^{25}$ already noted that a "mixed" conformation can be adopted by 5,10,15,20-tetrakis(3,5-tert-butyl-4-hydroxylphenyl) porphyrin (TDtBHPP) at the border of a hexagonal and a square packing of this molecule at a $\mathrm{Cu}(111)$ surface in UHV. They interpreted this phenomenon as a way how molecules can "accommodate a 2D lattice mismatch". Although the observations of Hill et al. are in line with our findings, it appears that for (TUP)Cu on the graphite/solvent interface the molecular conformations cause the different unit cell vectors, rather than mismatches in the monolayer lattices.

We propose that polymorphism with shared unit cell vectors might be a more general property in monolayers of adsorbates with conformational freedom of attached functional groups. This freedom can lead to shared unit cell vectors whenever part of a molecular conformation is shared between molecules adsorbed in different adjacent surface structures. This is expected to be more prevalent for molecules in which the conformation of each functional group is to a large extent independent from that of the other ones. For example, the rigidity of the porphyrin core of (TUP)Cu allows the alkyl tails to assume different conformations without affecting the conformation of the other tails. Similar conformational decoupling mechanisms might underlie the self-assembly behaviour of the HBC- $\mathrm{C}_{12}$ and bis-DBA derivatives mentioned above, in which the alkyl tails are also centred around rigid aromatic cores.

\section{Conclusion}

In this paper we have discussed the rich polymorphism of $2 \mathrm{D}$ surface structures of (TUP)Cu on graphite. STM images revealed four distinct 2-dimensional polymorphs (L, B, M, S), which were found to share unit cell vectors among each other. 
The presence of moire patterns in the STM images of the monolayers indicates that intermolecular interactions between the porphyrin adsorbates are a more predominant factor governing the structure of the adlayers than the interaction of the molecules with the underlying graphite. The layers are coincident with the graphite, implying that the substrate dictates preferred adsorption directions of the molecules, but not preferential adsorption positions. The different surface structures formed by (TUP)Cu connect in a seamless fashion, governed by the presence of shared unit cell vectors. We propose that partly shared conformations of the alkyl tails of molecules in adjacent polymorphs are the basis of these shared vectors. The relationship between molecular conformations and the shared unit cell vectors might be employed as a rational design parameter for self-assembled (multicomponent) monolayers. For the creation of functional devices it might be desired to connect regions of molecules with different function. The presence of shared unit cell vectors allows such connection in a stable, deterministic way, in contrast to adlayers of different species or different polymorphism for which the boundaries between different surface structures are stochastically formed and therefore difficult to control at the single molecule level.

\section{Experimental section}

Scanning Tunnelling Microscopy was performed on a home-built STM setup. ${ }^{26}$ STM images were flattened by first applying a plane fitting routine over the entire image and then subtracting the best fitting straight line from each individual scan line. No additional filtering was applied. Tips were mechanically cut from $0.5 \mathrm{~mm}$ diameter $\mathrm{Pt}_{0.8} \mathrm{Ir}_{0.2}$ wire and freshly cleaved $\mathrm{ZYB}$-grade Highly Oriented Pyrolytic Graphite (HOPG, NT-MDT) was used as a substrate. All measurements were performed at solid/liquid interfaces created by the application of a droplet of the solution under investigation between the tip and the substrate. The synthesis of TUP(Cu) has been described elsewhere. ${ }^{22}$ The solvents, 1-octanoic acid (98\%, Aldrich) and decamethyltetrasiloxane (97\%, Aldrich), were used as received.

\section{Acknowledgements}

NanoNed-the Dutch nanotechnology initiative by the Ministry of Economic Affairs is acknowledged for financial support. We thank the Australian Research Council for partial support (Project Grant DP120102254 to M.J.C. and J.R.R.). J.A.A.W.E. thanks the Council for the Chemical Sciences of the Netherlands Organization for Scientific Research (CW-NWO) for a Vidi grant (700.58.423), and the European Research Council for an ERC Starting Grant (NANOCAT - 259064).

\section{Notes and references}

1 C. A. Palma, M. Bonini, T. Breiner and P. Samorì, Adv. Mater., 2009, 21, 1383; J. V. Barth, G. Costantini and K. Kern, Nature, 2005, 437, 671.
2 S. Griessl, M. Lackinger, M. Edelwirth, M. Hietschold and W. M. Heckl, Single Mol., 2002, 3, 25; M. Lackinger, S. Griessl, W. A. Heckl, M. Hietschold and G. W. Flynn, Langmuir, 2005, 21, 4984; R. Gutzler, T. Sirtl, J. F. Dienstmaier, K. Mahata, W. M. Heckl, M. Schmittel and M. Lackinger, J. Am. Chem. Soc., 2010, 132, 5064.

3 S. Furukawa, H. Uji-i, K. Tahara, T. Ichikawa, M. Sonoda, F. C. De Schryver, Y. Tobe and S. De Feyter, J. Am. Chem. Soc., 2006, 128, 3502; K. Tahara, S. Furukawa, H. Uji-i, T. Uchino, T. Ichikawa, J. Zhang, W. Mamdouh, M. Sonoda, F. C. De Schryver, S. De Feyter and Y. Tobe, J. Am. Chem. Soc., 2006, 128, 16613; S. Lei, K. Tahara, F. C. De Schryver, M. Van der Auweraer, Y. Tobe and S. De Feyter, Angew. Chem., Int. Ed., 2008, 47, 2964; S. Lei, K. Tahara, Y. Tobe and S. De Feyter, Chem. Commun., 2010, 46, 9125; J. Adisoejoso, K. Tahara, S. Lei, P. Szabelski, W. Rzysko, K. Inukai, M. O. Blunt, Y. Tobe and S. De Feyter, ACS Nano, 2012, 6, 897.

4 G. Binnig and H. Rohrer, Helv. Phys. Acta, 1982, 55, 726.

5 J. A. A. W. Elemans, S. Lei and S. De Feyter, Angew. Chem., Int. Ed., 2009, 48, 7298.

6 K. Miyake, Y. Hori, T. Ikeda, M. Asakawa, T. Shimizu and S. Sasaki, Langmuir, 2008, 24, 4708.

7 P. Samorì, H. Engelkamp, P. de Witte, A. E. Rowan, R. J. M. Nolte and J. P. Rabe, Angew. Chem., Int. Ed., 2001, 40, 2348.

8 J. A. A. W. Elemans, M. C. Lensen, J. W. Gerritsen, H. van Kempen, S. Speller, R. J. M. Nolte and A. E. Rowan, Adv. Mater., 2003, 15, 2070.

9 S. Furukawa, K. Tahara, F. C. De Schryver, M. Van der Auweraer, Y. Tobe and S. De Feyter, Angew. Chem., Int. Ed., 2007, 46, 2831; S. Lei, K. Tahara, X. Feng, S. Furukawa, F. C. De Schryver, K. Müllen, Y. Tobe and S. De Feyter, J. Am. Chem. Soc., 2008, 130, 7119.

10 S. A. Burke, J. M. LeDue, J. M. Topple, S. Fostner and P. Grutter, Adv. Mater., 2009, 21, 2029.

11 J. Otsuki, Coord. Chem. Rev., 2010, 254, 2311.

12 X. Qiu, C. Wang, Q. Zeng, B. Xu, S. Yin, H. Wang, S. Xu and C. Bai, J. Am. Chem. Soc., 2000, 122, 5550; S. Lei, J. Wang, Y. H. Dong, C. Wang, L. J. Wan and C. L. Bai, Surf. Interface Anal., 2002, 34, 767; J. Otsuki, E. Nagamine, T. Kondo, K. Iwasaki, M. Asakawa and K. Miyake, J. Am. Chem. Soc., 2005, 127, 10400; B. Hulsken, R. van Hameren, J. W. Gerritsen, T. Khoury, P. Thordarson, M. J. Crossley, A. E. Rowan, R. J. M. Nolte, J. A. A. W. Elemans and S. Speller, Nat. Nanotechnol., 2007, 2, 285.

13 S. Yoshimoto, N. Yokoo, T. Fukuda, N. Kobayashi and K. Itaya, Chem. Commun., 2006, 500.

14 L. Grill, M. Dyer, L. Lafferentz, M. Persson, M. V. Peters and S. Hecht, Nat. Nanotechnol., 2007, 2, 687; S. Haq, F. Hanke, M. S. Dyer, M. Persson, P. Iavicoli, D. B. Amabilino and R. Raval, J. Am. Chem. Soc., 2011, 133, 12031; F. Hanke, S. Haq, R. Raval and M. Persson, ACS Nano, 2012, 5, 9093; L. Lafferentz, V. Eberhardt, C. Dri, C. Africh, G. Comelli, F. Esch, S. Hecht and L. Grill, Nat. Chem., 2012, 4, 215.

15 M. C. O’Sullivan, J. K. Sprafke, D. V. Kondratuk, C. Rinfray, T. D. W. Claridge, A. Saywell, M. O. Blunt, J. N. O'Shea, P. H. Beton, M. Malfois and H. Anderson, Nature, 2011, 469, 72. 
16 M. C. Lensen, J. A. A. W. Elemans, S. J. T. van Dingenen, J. W. Gerritsen, S. Speller, A. E. Rowan and R. J. M. Nolte, Chem.-Eur. J., 2007, 13, 7948.

17 M. J. J. Coenen, M. Cremers, D. den Boer, F. J. van den Bruele, T. Khoury, M. Sintic, M. J. Crossley, W. J. P. van Enckevort, B. L. M. Hendriksen, J. A. A. W. Elemans and S. Speller, Chem. Commun., 2011, 47, 9666.

18 A. Hoshino, S. Isoda, H. Kurata and T. Kobayashi, J. Appl. Phys., 1994, 76, 4113.

19 Y. Chin, D. Panduwinata, M. Sintic, T. J. Sum, N. S. Hush, M. J. Crossley and J. R. Reimers, J. Phys. Chem. Lett., 2011, 2, 62.

20 Q. Chen, H. J. Yan, C. J. Yan, G. P. Pan, L. J. Wan, G. Y. Wen and D. Q. Zhang, Surf. Sci., 2008, 602, 1256; M. E. Leunissen, W. S. Graswinckel, W. J. P. van Enckevort and E. Vlieg, Cryst. Growth Des., 2004, 4, 361.
21 G. Watel, F. Thibaudau and J. Cousty, Surf. Sci., 1993, 281, L297.

22 D. den Boer, T. Habets, M. J. J. Coenen, M. van der Maas, T. P. J. Peters, M. J. Crossley, T. Khoury, A. E. Rowan, R. J. M. Nolte, S. Speller and J. A. A. W. Elemans, Langmuir, 2011, 27, 2644.

23 L. Piot, A. Marchenko, J. Wu, K. Müllen and D. Fichou, J. Am. Chem. Soc., 2005, 127, 16245.

24 K. Tahara, S. Okuhata, J. Adisoejoso, S. Lei, T. Fujita, S. De Feyter and Y. Tobe, J. Am. Chem. Soc., 2009, 131, 17583.

25 J. P. Hill, Y. Wakayama and K. Ariga, Phys. Chem. Chem. Phys., 2006, 8, 5034.

26 J. W. Gerritsen, E. J. G. Boon, G. Janssens and H. van Kempen, Appl. Phys. A: Mater. Sci. Process., 1998, 66, S79. 